\title{
Standounkt
}

Alternative Kraftstoffe

\section{Kein Rettungsanker für unsere (Auto-)Mobilität}

\begin{abstract}
Alternative Kraftstoffe scheinen der optimale Weg zu weniger Schadstoffen im Straßenverkehr zu sein: viel fahren und den Treibhauseffekt trotzdem nicht verstärken. Oder doch nicht? Eine genaue Analyse zeigt die Begrenztheit von Biodiesel und Co. Von Stephan Ramesohl
\end{abstract}

$A^{\mathrm{ul}}$ lle Industrieländer suchen intensiv nach Wegen, die verkehrsbedingten Umweltbelastungen zu mindern und die Abhängigkeit vom Erdöl zu reduzieren. Als kurzfristige Optionen werden in erster Linie Bio-Kraftstoffe diskutiert, während langfristig vor allem Wasserstoff und Brennstoffzellen zur Erfüllung der Ziele beitragen sollen. Als Konsequenz ist zu beobachten, dass sich die Suche nach Alternativen im Straßenverkehr zunehmend auf die genannten Antriebstoffe konzentriert, ohne dass ein abgestimmter Systemansatz zu erkennen wäre.

Ob alternative Kraftstoffe die Umwelt weniger belasten, kann allerdings nicht alleine aus Sicht des Verkehrs beurteilt werden. Bewertungen müssen bei Substitution die Wechselwirkungen im gesamten Energiesystem berücksichtigen. Dies betrifft Fragen der Verfügbarkeit von Primärenergieträgern wie auch Effekte, die bei der Verlagerung von erneuerbaren Energien von stationären zu mobilen Anwendungen auftreten. Gerade im Bereich der Biokraftstoffe muss aus umweltpolitischer Sicht gefragt werden, ob das Ziel der EU-Biokraftstoffrichtlinie von 5,75 Prozent Biokraftstoff am Gesamtabsatz der Mitgliedsstaaten bis 2010 gegenwärtige Aktivitäten in eine sinnvolle Richtung lenkt.

\section{Flächenknappheit}

Im Moment sind nur die Biokraftstoffe Biodiesel und Ethanol in größeren Mengen marktverfügbar, sodass die poli- tische Förderung in erster Linie den Ausbau dieser Routen forciert. Die Verwendung von Flächen für den Energiepflanzenanbau steht dabei unter anderem in Konkurrenz zum Naturschutz. Unter heutigen Bedingungen können die Zielwerte der Richtlinie nur unter Verletzung von Naturschutzkriterien erreicht werden, da erst mittel- bis langfristig die verfügbare ökologisch-tragfähige Anbaufläche etwa durch Flächenstilllegungen oder Rückgang der heimischen Nahrungsmittelproduktion zunimmt.

Die derzeitige Fokussierung auf Biodiesel und Bioethanol vernachlässigt andere Möglichkeiten, die deutlich höhere Flächenerträge erwarten lassen. Vor allem der Anbau von Energiepflanzen als Substrat für Biogasanlagen verspricht, verglichen mit dem Rapsanbau, bis zu dreimal höhere Energieerträge pro Hektar. Der schrittweise Ausbau von Erdgastankstellen ermöglicht, in Zukunft verstärkt Biogas aufzubereiten und ins Erdgasnetz für mobile Nutzungen einzuspeisen.

Grundsätzlich gilt unter deutschen Bedingungen, dass stationäre Biomasseoptionen zur Stromerzeugung durch die Verdrängung von Kohlestrom einen signifikant höheren Klimaschutzbeitrag leisten als die Substitution von Benzin und Diesel im Verkehr. Dies ist auch für das große Potenzial der holzartigen Reststoffe relevant, für die gegenwärtig die Vergasung und Synthese zu Diesel unter dem Stichwort Biomass-to-Liquid diskutiert wird. In stationären Anwendungen zur Strom- und Wärmeerzeugung kann ein 50 bis 100 Prozent höherer Reduktionseffekt bei Kohlendioxid erzielt werden und gleichzeitig zur fälligen Erneuerung des Kraftwerksparks in Europa beitragen.

\section{Technologiekonkurrenz}

Vergleichbares gilt für die Bereitstellung von Wasserstoff auf Basis erneuerbarer Energien, da hier ebenfalls die Wasserstofferzeugung in unmittelbarer Konkurrenz zur direkten Nutzung der erneuerbaren Energie im Strom- und Wärmesystem steht. Als Konsequenz sollte sich die Energie-, Verkehrs- und Umweltpolitik gemeinsam bemühen, möglichst sinnvolle und effiziente Verwendungsrouten für die erneuerbaren Energiequellen zu finden und ihren Nutzen zu optimieren.

Da erneuerbare Energien kurz- bis mittelfristig nur begrenzt verfügbar sind und ihr Ausbau nicht beliebig beschleunigt werden kann, bleibt die nüchterne Feststellung, dass alternative Kraftstoffe nur einen Teilbeitrag leisten können. Um eine ökologisch wie ökonomisch nachhaltige Mobilität im Straßenverkehr sicherzustellen, führt kein Weg an der Steigerung der Energieeffizienz vorbei. Oft wurde sie beschworen, gerne verdrängt und immer wieder von Wirtschaft und Politik vernachlässigt. Und dennoch gilt: Angesichts der Wachstumsdynamik ist eine drastische Senkung der Energieverbräuche der Fahrzeuge die unverzichtbare Voraussetzung, um signifikante Deckungsanteile von alternativen Kraftstoffen überhaupt erst zu ermöglichen. $\leftarrow$

\section{AUTOR + KONTAKT}

Dr. Stephan Ramesohl ist Mitarbeiter der Forschungsgruppe Zukünftige Energieund Mobilitätsstrukturen am Wuppertal Institut für Klima, Umwelt, Energie (WI).

WI, Postfach 1004 80, 42004 Wuppertal. Tel. 0202/2492 -255, E-Mail: stephan.ramesohl@wupperinst.org 
(c) 20I0 Authors; licensee IÖW and oekom verlag. This is an article distributed under the terms of the Creative Commons Attribution Non-Commercial No Derivates License (http://creativecommons.org/licenses/by-nc-nd/3.o/), which permits unrestricted use, distribution, and reproduction in any medium, provided the original work is properly cited. 